Proceedings of the 2007 Winter Simulation Conference

S. G. Henderson, B. Biller, M.-H. Hsieh, J. Shortle, J. D. Tew, and R. R. Barton, eds.

\title{
SINGLE-STAGE MULTIPLE-COMPARISON PROCEDURE FOR QUANTILES AND OTHER PARAMETERS
}

\author{
Marvin K. Nakayama \\ Computer Science Department \\ New Jersey Institute of Technology \\ Newark, N.J. 07102, U.S.A.
}

\begin{abstract}
We present a single-stage multiple-comparison procedure for comparing parameters of independent systems, where the parameters are not necessarily means or steady-state means. We assume that for each system, the parameter has an estimation process that satisfies a central limit theorem (CLT) and that we have a consistent variance-estimation process for the variance parameter appearing in the CLT. The procedure allows for unequal run lengths or sample sizes across systems, and also allows for unequal and unknown variance parameters across systems. The procedure is asymptotically valid as the run lengths or sample sizes of all system grow large. One setting the framework encompasses is comparing quantiles of independent populations. It also covers comparing means or other moments of independent populations, functions of means, and steady-state means of stochastic processes.
\end{abstract}

\section{INTRODUCTION}

Suppose that we have $k$ systems, labeled $1,2, \ldots, k$, that are to be compared relative to a given parameter. Let $\theta_{i}$ denote the value of the parameter for system $i$. For example, suppose there are 10 possible designs for a fault-tolerant computing system, and we want to compare the alternatives in terms of their 0.9-quantile of the time to failure.

This paper presents a single-stage multiple-comparison procedure to compare $\theta_{1}, \theta_{2}, \ldots, \theta_{k}$, where we assume larger values of $\theta_{i}$ are better. Specifically, we consider multiple comparisons with the best (MCB, Hsu 1984), which produces simultaneous confidence intervals for $\theta_{i}-\max _{j \neq i} \theta_{j}$ for $i=1,2, \ldots, k$. Note that if $\theta_{i}-\max _{j \neq i} \theta_{j}>0$, then system $i$ is the best. In some situations when secondary considerations (e.g., ease of installation) are taken into account, one may opt to implement a non-optimal design, as long as it is "close enough" to the best. For example, we may choose a system $i$ with $\theta_{i}-\max _{j \neq i} \theta_{j}<0$ as long as $\theta_{i}-\max _{j \neq i} \theta_{j}>-\delta$ for some specified $\delta>0$, so system $i$ is not the best but is within $\delta$ of the best.

As the run length or sample size of each system grows to infinity, our MCB procedure is asymptotically valid under the following two assumptions. First we assume that for each system $i, \theta_{i}$ has an estimation process that satisfies a central limit theorem (CLT). The CLT has a variance parameter $\sigma_{i}^{2}$, and we require that we have a consistent estimator for $\sigma_{i}^{2}$. We allow for the run lengths or sample sizes of the different systems to differ, and we allow for the $\sigma_{i}^{2}, i=1,2, \ldots, k$, to be unequal and unknown.

Most previous work on multiple-comparison procedures (Hochberg and Tamhane 1987, Swisher, Jacobson, and Yucesan 2003, Kim and Nelson 2006) assumes that $\theta_{i}$ is the mean of a normally distributed population, and that independent and identically distributed (i.i.d.) sampling is used within each population. Exceptions include Nakayama (1997) and Damerdji and Nakayama (1999), which compare steadystate means using standardized time series (STS) methods (Schruben 1983, Glynn and Iglehart 1990) for "estimating" $\sigma_{i}^{2}$. STS methods do not yield consistent estimates of the $\sigma_{i}^{2}$ when the run length grows large but the number of batches remains fixed, so the results in the current paper do not include the multiple-comparison methods using STS.

Our framework in the current paper allows for comparing systems relative to quite general parameters, including means or other moments of independent (not necessarily normally distributed) populations, functions of means, and steady-state means of stochastic processes. One specific example we consider here is comparing quantiles of independent populations.

The rest of the paper is organized as follows. Section 2 describes the mathematical framework we adopt. We present our MCB procedure in Section 3, and Section 4 shows explicitly how the procedure applies to comparing quantiles of independent populations. Section 5 presents empirical results comparing quantiles, and we summarize our findings in Section 6. 


\section{MATHEMATICAL FRAMEWORK}

Suppose that we have $k$ systems, labeled $1,2, \ldots, k$, where the $i$ th system has parameter $\theta_{i}$. The goal is to compare the $k$ systems in terms of the $\theta_{i}$. We assume that for each $i$, we have an estimation process $\widehat{\theta}_{i}=\left[\widehat{\theta}_{i}(t): t>0\right]$ for $\theta_{i}$, where $\widehat{\theta}_{i}(t)$ is the estimator of $\theta_{i}$ based on running a simulation of system $i$ for a run length of $t$ or taking $t$ i.i.d. samples from population $i$, depending on the context. Let $N(a, b)$ denote a normal random variable with mean $a$ and variance $b$, and let $\Rightarrow$ denote weak convergence (see Chapter 5 of Billingsley 1995). We assume that each $\widehat{\theta}_{i}$ satisfies a central limit theorem:

Assumption 1 The estimation processes $\widehat{\theta}_{1}, \ldots, \widehat{\theta}_{k}$ are independent, and there exists a finite positive constant $\eta$ such that for each $i$,

$$
t^{\eta}\left[\widehat{\theta}_{i}(t)-\theta_{i}\right] \Rightarrow N\left(0, \sigma_{i}^{2}\right)
$$

as $t \rightarrow \infty$, where $0<\sigma_{i}<\infty$ is a constant.

In most applications, the parameter $\eta$ in Assumption 1 takes on the canonical value of $1 / 2$, but we do not require this. Glynn and Whitt (1992) list examples of processes satisfying the CLT in (1). For example, the CLT holds (under various conditions) for sample means or other sample moments of i.i.d. samples, functions of sample means, quantile estimators, and time-average rewards of stochastic processes having a steady state. Specifically, for the last example, let $X_{i}=\left[X_{i}(t): t \geq 0\right]$ be a stochastic process on state space $S_{i}$ representing the evolution over time of system $i$, and let $f_{i}: S_{i} \rightarrow \Re$ be a real-valued "reward" function on $S_{i}$. Then we can define $\widehat{\theta}_{i}(t)=(1 / t) \int_{0}^{t} f_{i}\left(X_{i}(s)\right) d s$, which is the time-average reward of $X_{i}$ over the interval $[0, t]$. Under a wide variety of assumptions (Glynn and Iglehart 1990), $\widehat{\theta}_{i}$ satisfies the CLT in (1) with $\eta=1 / 2$, and $\theta_{i}$ is the steady-state mean reward of $X_{i}$.

We call $\sigma_{i}^{2}$ in (1) the variance parameter for system $i$. We assume that for each $i$, we have a variance-estimation process $V_{i}=\left[V_{i}(t): t>0\right]$ that is consistent, in the following sense: $t \rightarrow \infty$.

Assumption 2 For each system $i, V_{i}(t) \Rightarrow \sigma_{i}^{2}$ as

In our previous example of comparing steady-state mean rewards, suppose that each process $X_{i}$ is regenerative (Crane and Iglehart 1975), and let $0 \leq A_{i, 0}<A_{i, 1}<A_{i, 2}<\cdots$ be the sequence of regeneration epochs of system $i$. For $j=1,2, \ldots$, let $\tau_{i, j}=A_{i, j}-A_{i, j-1}$ be the length of the $j$ th cycle of system $i$. Also, define $Y_{i, j}=\int_{A_{i, j-1}}^{A_{i, j}} f_{i}\left(X_{i}(s)\right) d s$ to be the cumulative reward over the $j$ th cycle of system $i$. Assume that $E\left[\tau_{i, 1}\right]<\infty$ and that there exists a finite constant $\theta_{i}$ such that $E\left[Y_{i, 1}-\theta_{i} \tau_{i, 1}\right]=0$ and $E\left[\left(Y_{i, 1}-\theta_{i} \tau_{i, 1}\right)^{2}\right]<\infty$. Then $\theta_{i}=E\left[Y_{i, 1}\right] / E\left[\tau_{i, 1}\right]$ and the CLT in (1) holds with $\eta=1 / 2$ and $\sigma_{i}^{2}=E\left[\left(Y_{i, 1}-\theta_{i} \tau_{i, 1}\right)^{2}\right] / E\left[\tau_{i, 1}\right]$. Define $N_{i}(t)=\sup \{j \geq$ $\left.0: A_{i, j} \leq t\right\}$, which is the number of regenerative cycles that process $i$ completes by time $t$. Then the variance estimator

$$
V_{i}(t)=\frac{1}{t} \sum_{j=1}^{N_{i}(t)}\left[Y_{i, j}-\widehat{\theta}_{i}(t) \tau_{i, j}\right]^{2}
$$

satisfies Assumption 2; see Glynn and Iglehart (1993) for details.

An example of a setting in which Assumption 1 holds for a non-canonical value of $\eta$ is for estimators based on the Kiefer-Wolfowitz (1952) stochastic approximation algorithm. Ruppert (1982) shows that under certain regularity conditions, such estimators satisfy (1) with $\eta=1 / 3$. Ventner (1967), p. 189, provides directions for constructing $V_{i}(t)$ such that Assumption 2 holds.

\section{MCB PROCEDURE}

We now present a MCB procedure for comparing the parameters $\theta_{i}, i=1,2, \ldots, k$, of independent systems.

1. Specify the number of systems $2 \leq k<\infty$, the confidence level $1-\alpha$ with $0<\alpha<1$, and $\bar{T}=\left(T_{1}, \ldots, T_{k}\right)$, where $T_{i}$ is the run length (or sample size) for system $i$. Define the constant $\gamma=z_{(1-\alpha)^{1 /(k-1)}}$, where $z_{\beta}$ satisfies $\Phi\left(z_{\beta}\right)=\beta$ for $0<\beta<1$, and $\Phi$ is the distribution function of a standard (mean 0 and variance 1 ) normal distribution.

2. Run independent simulations of the $k$ systems, where the simulation of system $i$ has a run length of $T_{i}$.

3. For each system $i$, compute $V_{i}\left(T_{i}\right)$, and define the joint MCB intervals $I_{i}(\bar{T}), i=1,2, \ldots, k$, for $\theta_{i}-\max _{j \neq i} \theta_{j}, i=1,2, \ldots, k$, respectively, with

$I_{i}(\bar{T})$

$$
\begin{gathered}
=\left[-\left(\min _{\substack{j \in \mathscr{A}(\bar{T}), j \neq i}}\left(\widehat{\theta}_{i}\left(T_{i}\right)-\widehat{\theta}_{j}\left(T_{j}\right)-W_{i, j}(\bar{T})\right)\right)^{-},\right. \\
\left.\left(\min _{j \neq i}\left(\widehat{\theta}_{i}\left(T_{i}\right)-\widehat{\theta}_{j}\left(T_{j}\right)+W_{i, j}(\bar{T})\right)\right)^{+}\right],
\end{gathered}
$$

where $(x)^{+}=\max (x, 0),-(x)^{-}=\min (x, 0)$,

$$
W_{i, j}(\bar{T})=\gamma \sqrt{\frac{V_{i}\left(T_{i}\right)}{T_{i}^{2 \eta}}+\frac{V_{j}\left(T_{j}\right)}{T_{j}^{2 \eta}}},
$$

and

$\mathscr{A}(\bar{T})=\left\{\ell: \min _{j \neq \ell}\left(\widehat{\theta}_{\ell}\left(T_{\ell}\right)-\widehat{\theta}_{j}\left(T_{j}\right)+W_{\ell, j}(\bar{T})\right) \geq 0\right\}$.

In (2), we define $\min _{j \in \emptyset} x_{j}=0$. 


\section{Nakayama}

The following establishes the asymptotic validity of the MCB intervals in (2).

Theorem 1 Suppose $T_{i}=\zeta_{i} T$ for $i=1,2, \ldots, k$, where $\zeta_{1}>0, \ldots, \zeta_{k}>0$, are any constants. If Assumptions 1 and 2 hold, then

$$
\lim _{T \rightarrow \infty} P\left\{\theta_{i}-\max _{j \neq i} \theta_{j} \in I_{i}(\bar{T}), i=1,2, \ldots, k\right\} \geq 1-\alpha .
$$

\section{QUANTILES}

We now specifically discuss the setting of comparing quantiles of independent populations, which covers the case of comparing quantiles in terminating simulations. For example, given $k$ alternative designs for a manufacturing system that shuts down each night, we might be interested in comparing the alternatives in terms of the 0.9-quantiles of the average flow times of the first 10 jobs each day.

For $0<y<1$ and any distribution function $G$, we define the $y$ th quantile of $G$ to be $G^{-1}(y) \equiv \inf \{x: G(x) \geq y\}$. Let $F_{i}$ be the distribution function of population $i$. Fix $p$ with $0<p<1$, and let $\theta_{i}=F_{i}^{-1}(p)$ be the $p$ th quantile of system $i$. For each system $i$, let $X_{i, 1}, X_{i, 2}, \ldots, X_{i, t}$ be $t$ i.i.d. samples from $F_{i}$, and define the empirical distribution function $F_{i, t}(x)=(1 / t) \sum_{j=1}^{t} 1\left\{X_{i, j} \leq x\right\}$, where $1\{A\}$ is the indicator function of the event $A$. Also, define

$$
\widehat{\theta}_{i}(t)=F_{i, t}^{-1}(p)
$$

the estimator of $\theta_{i}$ based on $t$ samples from $F_{i}$.

Assume that for each $i, F_{i}$ is differentiable at $\theta_{i}$, and $F_{i}^{\prime}\left(\theta_{i}\right)>0$, where prime denotes derivative. Then the CLT in Assumption 1 holds with $\eta=1 / 2$ and

$$
\sigma_{i}^{2}=\frac{p(1-p)}{\left[F_{i}^{\prime}\left(\theta_{i}\right)\right]^{2}}
$$

e.g., see p. 77 of Serfling (1980). To develop an estimator for $\sigma_{i}^{2}$, define constants $q_{i, t}$ such that

$$
q_{i, t}=p+\sqrt{\frac{p(1-p)}{t}}+o\left(\frac{1}{t^{1 / 2}}\right)
$$

as $t \rightarrow \infty$, where $f(t)=o(g(t))$ for functions $f$ and $g$ means $f(t) / g(t) \rightarrow 0$ as $t \rightarrow \infty$. Then the variance estimator

$$
V_{i}(t)=t\left[F_{i, t}^{-1}\left(q_{i, t}\right)-F_{i, t}^{-1}(p)\right]^{2}
$$

satisfies Assumption 2; e.g., see p. 94 of Serfling (1980). Thus, we can apply the MCB procedure in Section 3 with $\widehat{\theta}_{i}(t)$ and $V_{i}(t)$ defined in (3) and (6), respectively. In the current setting of comparing quantiles, $T_{i}$ in the MCB procedure of Section 3 denotes the number of i.i.d. samples taken from population $i$.

\section{EMPIRICAL RESULTS}

We ran some experiments with our MCB procedure to compare the $p$ th quantiles of independent populations. Specifically, we compared $k=4$ exponentially distributed populations, where the $i$ th population has distribution function $F_{i}(x)=1-e^{-x / \mu_{i}}$ for $x \geq 0$, so its mean is $\mu_{i}$. In all our experiments, we fixed $\mu_{1}=\mu_{2}=\mu_{3}=1$, and we varied $\mu_{4}$ over the values 1.1, 1.2, 2 and 5. Thus, the $p$ th quantile of the $i$ th population is $\theta_{i}=-\mu_{i} \ln (1-p)$, which we assume is unknown, and for any fixed $p$, population 4 is the best since $\theta_{4}$ is the largest among $\theta_{1}, \ldots, \theta_{4}$, which we also assume is unknown. We used the same sample size $T_{1}=\cdots=T_{4}=T$ for each population, and we set $T$ to be 20,80 or 320 . We varied the quantile level $p$ between 0.1 and 0.9. From (4) we can calculate $\sigma_{i}^{2}=\mu_{i}^{2} p /(1-p)$, which we assume is unknown, and we estimated $\sigma_{i}^{2}$ in our experiments using $V_{i}(T)$ in (6) with $q_{i, T}=p+\sqrt{p(1-p) / T}$ in (5). We ran $10^{4}$ independent replications for each set of parameters, where we constructed MCB intervals having nominal confidence level $1-\alpha=0.9$ in each replication.

Table 1 gives the coverage results from our simulations. In all but one case, for a fixed sample size $T$ and quantile level $p$, the coverage increases as $\mu_{4}$ increases, which also corresponds to $\theta_{4}-\theta_{i}, i=1,2,3$, increasing. Moreover, for a fixed configuration of means and quantile level $p$, coverage increases as the sample size $T$ for each population increases (i.e., as we go across a row), with the coverage levels close to or at the nominal level of $90 \%$ for the largest sample size $T=320$. This agrees with our asymptotic theory in Theorem 1. In addition, considering the coverages for all $p$ and means for each sample size $T$, we see that there is a larger range of coverages for smaller $T$ than for larger $T$. This may indicate that quantile estimators and their variance estimators require large sample sizes to be accurate. Finally, comparing the coverages for the different quantile levels $p$ for the same configuration of means and for same $T$, we see that the coverages are typically lower for $p=0.1$ and 0.9 than for $p=0.3,0.5$ or 0.7 when $T$ is either 20 or 80 , which is when coverages are significantly below the nominal level of 0.9. This may indicate that extreme quantiles (i.e., when $p$ is close to 0 or 1 ) are harder to estimate than those with $p$ close to 0.5 .

For comparison Table 2 presents results from running experiments comparing the means of the 4 exponential distributions, so now each parameter $\theta_{i}=\mu_{i}$. In this case the coverages are all close to the nominal level of $1-\alpha=0.9$, even for the smallest sample size $T=20$. Hence, the asymptotics for MCB intervals comparing means seem to take effect for smaller sample sizes than they do when comparing quantiles. 
Table 1: Coverage results (in percents) for MCB intervals comparing $p$ th quantiles of $k=4$ exponential populations, with $T$ samples from each population.

\begin{tabular}{c|l|ccc}
\hline & & \multicolumn{3}{|c}{$T$} \\
\cline { 3 - 5 }$p$ & Means & 20 & 80 & 320 \\
\hline \hline 0.1 & $1,1,1,1.1$ & 72.4 & 75.7 & 86.3 \\
0.1 & $1,1,1,1.2$ & 72.6 & 76.1 & 86.7 \\
0.1 & $1,1,1,2$ & 73.9 & 78.8 & 88.3 \\
0.1 & $1,1,1,5$ & 75.7 & 81.6 & 90.1 \\
\hline 0.3 & $1,1,1,1.1$ & 84.9 & 86.8 & 89.3 \\
0.3 & $1,1,1,1.2$ & 85.1 & 87.1 & 89.5 \\
0.3 & $1,1,1,2$ & 86.1 & 88.0 & 90.7 \\
0.3 & $1,1,1,5$ & 87.2 & 89.6 & 92.4 \\
\hline 0.5 & $1,1,1,1.1$ & 83.6 & 85.3 & 86.7 \\
0.5 & $1,1,1,1.2$ & 83.8 & 85.7 & 87.0 \\
0.5 & $1,1,1,2$ & 84.7 & 86.6 & 88.7 \\
0.5 & $1,1,1,5$ & 85.3 & 88.4 & 90.6 \\
\hline 0.7 & $1,1,1,1.1$ & 86.7 & 89.1 & 90.2 \\
0.7 & $1,1,1,1.2$ & 86.6 & 89.2 & 90.5 \\
0.7 & $1,1,1,2$ & 86.9 & 89.8 & 91.4 \\
0.7 & $1,1,1,5$ & 87.0 & 90.9 & 92.4 \\
\hline 0.9 & $1,1,1,1.1$ & 74.9 & 78.2 & 88.6 \\
0.9 & $1,1,1,1.2$ & 74.8 & 78.3 & 88.9 \\
0.9 & $1,1,1,2$ & 73.8 & 79.7 & 89.6 \\
0.9 & $1,1,1,5$ & 72.8 & 80.3 & 90.8 \\
\hline
\end{tabular}

\section{CONCLUSIONS}

We presented a single-stage MCB procedure for comparing parameters $\theta_{1}, \ldots, \theta_{k}$ of independent systems. The procedure is asymptotically valid when the estimator of each $\theta_{i}$ satisfies a CLT and there is a consistent estimator of the variance parameter appearing in the CLT. The procedure allows for unequal run lengths across systems, and unknown and unequal variances. This framework encompasses comparing means of populations, functions of means, quantiles, and steady-state means.

We consider two-stage MCB procedures in Nakayama (2006) for the same general framework as in the current paper. The two-stage procedures have random run lengths for each system, and to establish the asymptotic validity of these procedures, we require a slight strengthening of the CLT assumption so that the CLT also holds when applied at the random time.

\section{REFERENCES}

Billingsley, P. 1995. Probability and measure. Third ed. New York: John Wiley \& Sons.

Crane, M., and D. L. Iglehart. 1975. Simulating stable stochastic systems, III: Regenerative processes and
Table 2: Coverage results (in percents) for MCB intervals comparing the means of $k=4$ exponential populations, with $T$ samples from each population.

\begin{tabular}{l|ccc}
\hline \multirow{2}{*}{ Means } & \multicolumn{3}{|c}{$T$} \\
\cline { 2 - 4 } & 20 & 80 & 320 \\
\hline \hline $1,1,1,1.1$ & 91.3 & 91.6 & 91.7 \\
$1,1,1,1.2$ & 90.9 & 91.4 & 91.7 \\
$1,1,1,2$ & 88.9 & 91.0 & 92.4 \\
$1,1,1,5$ & 88.6 & 92.0 & 93.9 \\
\hline
\end{tabular}

discrete-event simulations. Operations Research 23:3345.

Damerdji, H., and M. K. Nakayama. 1999. Two-stage multiple-comparison procedures for steady-state simulations. ACM Transactions on Modeling and Computer Simulations 9:1-30.

Glynn, P. W., and D. L. Iglehart. 1990. Simulation output analysis using standardized time series. Mathematics of Operations Research 15:1-16.

Glynn, P. W., and D. L. Iglehart. 1993. Conditions for the applicability of the regenerative method. Management Science 39:1108-1111.

Glynn, P. W., and W. Whitt. 1992. The asymptotic validity of sequential stopping rules in stochastic simulations. Annals of Applied Probability 2:180-198.

Hochberg, Y., and A. C. Tamhane. 1987. Multiple comparison procedures. New York: Wiley.

Hsu, J. C. 1984. Constrained simultaneous confidence intervals for multiple comparisons with the best. Annals of Statistics 12:1136-1144.

Kiefer, J., and J. Wolfowitz. 1952. Stochastic estimation of the maximum of a regression function. Annals of Mathematical Statistics 23:462-466.

Kim, S.-H., and B. L. Nelson. 2006. Selecting the best system. In Elsevier Handbooks in Operations Research and Management Science: Simulation. Amsterdam: Elsevier.

Nakayama, M. K. 1997. Multiple-comparison procedures for steady-state simulations. Annals of Statistics 25:24332450.

Nakayama, M. K. 2006. Two-stage selection and multiplecomparison procedures for simulations with weakly consistent variance estimators. In preparation.

Ruppert, D. 1982. Almost sure approximations to the Robbins-Munro and Kiefer-Wolfowitz processes with dependent noise. Annals of Probability 10:178-187.

Schruben, L. W. 1983. Confidence interval estimation using standardized time series. Operations Research 31:10901108.

Serfling, R. J. 1980. Approximation theorems of mathematical statistics. New York: John Wiley \& Sons. 
Swisher, J. R., S. H. Jacobson, and E. Yucesan. 2003. Discrete-event simulation optimization using ranking, selection, and multiple comparison procedures: A survey. ACM Transactions on Modeling and Computer Simulation 13:134-154.

Ventner, J. H. 1967. An extension of the Robbins-Munro procedure. Annals of Mathematical Statistics 38:181190.

\section{AUTHOR BIOGRAPHY}

MARVIN K. NAKAYAMA is an associate professor in the Department of Computer Science at the New Jersey Institute of Technology. He received a Ph.D. in operations research from Stanford University. He won second prize in the 1992 George E. Nicholson Student Paper Competition sponsored by INFORMS and is a recipient of a CAREER Award from the National Science Foundation. He is the stochastic models area editor for ACM Transactions on Modeling and Computer Simulation and the simulation are editor for INFORMS Journal on Computing. His research interests include applied probability, statistics, simulation and modeling. His e-mail address is $\operatorname{marvinenjit.~}$ edu , and his web page is $\langle$ web.njit.edu/ marvin〉. 